\title{
Galectin-1 is a poor prognostic factor in patients with glioblastoma multiforme after radiotherapy
}

\author{
Shang-Yu Chou', Shao-Lun Yen ${ }^{5}$, Chao-Cheng Huang ${ }^{2,4}$ and Eng-Yen Huang ${ }^{1,3,4,6^{*}}$ (D)
}

\begin{abstract}
Background: Galectin-1, a radioresistance marker, was found in our previous study to be a prognostic factor for cervical cancer. The aim of current study is to determine the prognostic significance of the galectin-1 expression level in patients with glioblastoma multiforme (GBM) undergoing adjuvant radiotherapy (RT).

Methods: We included 45 patients with GBM who were treated with maximal safe surgical resection or biopsy alone followed by adjuvant RT of EQD2 (equivalent dose in 2-Gy fractions) > or $=60$ Gy for homogeneous treatment. Paraffin-embedded tissues acquired from the Department of Pathology were analyzed using immunohistochemical staining for galectin-1 expression. The primary endpoint was overall survival (OS).

Results: Patients with weak expression had a better median survival (27.9 months) than did those with strong expression (10.7 months; $p=0.009$ ). We compared characteristics between weak and strong galectin-1 expression, and only the expression level of galectin-3 showed a correlation. The group with weak galectin-1 expression displayed a 3-year OS of $27.3 \%$ and a 3 -year cancer-specific survival (CSS) of $27.3 \%$; these values were only $5.9 \%$ and $7.6 \%$, respectively, in the group with strong galectin-1 expression ( $p=0.009$ and 0.020 , respectively). Cox regression was used to confirm that the expression level of galectin-1 (weak vs. strong) is a significant factor of OS ( $p=0.020)$ and CSS $(p=0.022)$. Other parameters, such as the expression level of galectin-3, Eastern Cooperative Oncology Group (ECOG) performance, gender, surgical method, age $\geq 50$ years, tumor size, or radiation field were not significant factors.
\end{abstract}

Conclusion: The expression level of galectin-1 affects survival in patients with GBM treated with adjuvant RT. Future studies are required to analyze the effect of other factors, such as $\mathrm{O}(6)$-methylguanine-DNA methyltransferase (MGMT)-promoter methylation status, in patients with weak and strong galectin-1 expression.

Keywords: Galectin-1, Glioblastoma multiforme, Adjuvant radiotherapy, Radioresistant marker

\section{Background}

Glioblastoma multiforme (GBM) is the most common type of brain tumor, accounting for $60 \%$ of all malignant primary brain tumors in adults; GBM is also the most malignant type. Although GBM is rare, with an incidence of $2-3$ cases per 100,000 patients with primary malignant brain tumors in Europe and North America [1], a significant increase in the incidence of GBM has been observed [2]. In general, the disease progresses

\footnotetext{
* Correspondence: huangengyen@gmail.com

'Departments of Radiation Oncology, Kaohsiung Chang Gung Memorial Hospital, 123 Ta-Pei Road, Niao-Song Dist, Kaohsiung City 83301, Taiwan ${ }^{3}$ Department of Radiation Oncology, Xiamen Chang Gung Hospital, No. 123, Xiafei Rd., Haicang District, Fujian, China

Full list of author information is available at the end of the article
}

rapidly and has a poor outcome. Without treatment, overall survival (OS) in GBM patients is only $3-5$ months [3], and despite multimodal aggressive treatment, the median survival of GBM is still only 12 months. Currently, maximal safe surgical resection followed by adjuvant local radiotherapy (RT) and temozolomide (TMZ) is considered the standard treatment for GBM. In 2005, a large clinical trial of 575 participants randomized to treatment with standard RT vs. RT plus TMZ chemotherapy reported that the latter group survived a median of 14.6 months, as opposed to 12.1 months for the former group [4]. Nonetheless, the effectiveness of TMZ is weak in patients without $\mathrm{O}(6)$-methylguanine-DNA methyltransferase (MGMT) promoter methylation [5]. 
Another possible approach is to target other molecules that can combine with radiation sensitizers to increase the therapeutic effect.

Galectin-1, a lectin that binds to the galactoside moiety of glycoprotein, has been associated with GBM progression via processes of migration [6], invasion [6, 7], angiogenesis [8, 9], and immune escape [10]. We first found galectin-1 to be involved in radioresistance in cervical cancer [11, 12]. However, in contrast to cervical cancer, GBM exhibits high galectin-1 expression [13, 14] and is radioresistant $[15,16]$. Therefore, in this study, we sought to investigate role of galectin-1 in determining the outcomes of patients undergoing RT alone without planned concomitant nor adjuvant chemotherapy. The results confirmed our hypothesis that GBM patients with strong galectin-1 expression have poor OS.

\section{Methods}

\section{Patients' characteristics}

This study was approved by the Institutional Review Board of Chang Gung Memorial Hospital (102-5087B). A total of 63 newly diagnosed GBM patients who were treated at our institution before January 1, 2002, were retrospectively evaluated. Different radiation doses may affect prognosis in GBM patients; Bleehen et al. reported that a survival advantage for patients with grades 3 and 4 astrocytoma was maintained with the high dose of 60 Gy [17]. The current standard dose for GBM at our institution is also $60 \mathrm{~Gy}$, which is according to NCCN guidelines. Hence, we excluded patients with an equivalent dose in 2-Gy fractions (EQD2) < 60 Gy for homogeneous treatment due to poor outcome [17] to exclude the dose effect, which might mask the importance of galectin-1 for prognosis, and selected those with EQD2 $\geq$ $60 \mathrm{~Gy}$. A total of 45 patients remained and were treated with maximal safe surgical resection or biopsy only followed by adjuvant RT of EQD2 $\geq 60$ Gy for homogeneous treatment; the outcomes of these patients were analyzed (Table 1 ). We chose age $\geq 50$ years as a cutoff based on a recursive partitioning analysis (RPA) study [18]; we chose a tumor size $\geq 5 \mathrm{~cm}$ as a cutoff because this size is a significant prognostic factor after RT [19]. Brain image analysis by magnetic resonance imaging (MRI) or computed tomography (CT) was performed before treatment. The tumor size was measured in the longest diameter on a T1-weighted axial contrastenhanced image by brain MRI or axial contrastenhanced image by brain CT. For multifocal GBM, the sum of the largest axial diameter for all lesions was used to determine the tumor size.

\section{Radiation therapy}

Patients were assigned by the radiation oncologist either for whole-brain irradiation (19 patients) followed by
Table 1 Patient characteristics $(n=45)$

\begin{tabular}{|c|c|c|c|}
\hline Parameter & $\begin{array}{l}\text { Gal-1 expression } \\
\text { (weak) }\end{array}$ & $\begin{array}{l}\text { Gal-1 expression } \\
\text { (strong) }\end{array}$ & $p$ value \\
\hline Age (years) & & & 0.464 \\
\hline$<50$ & $5(45.5 \%)$ & $10(29.4 \%)$ & \\
\hline$\geq 50$ & $6(54.5 \%)$ & $24(70.6 \%)$ & \\
\hline Gender & & & 0.732 \\
\hline Female & $5(45.5 \%)$ & $13(38.2 \%)$ & \\
\hline Male & $6(54.5 \%)$ & $21(61.8 \%)$ & \\
\hline ECOG & & & 1.000 \\
\hline $0-2$ & 8 (72.7\%) & $26(76.5 \%)$ & \\
\hline $3-4$ & $3(27.3 \%)$ & $8(23.5 \%)$ & \\
\hline Tumor size & & & 0.897 \\
\hline$<5 \mathrm{~cm}$ & $3(27.3 \%)$ & 7 (20.6\%) & \\
\hline$\geq 5 \mathrm{~cm}$ & $2(18.2 \%)$ & 7 (20.6\%) & \\
\hline Unknown & $6(54.5 \%)$ & $20(58.8 \%)$ & \\
\hline OP method & & & 0.525 \\
\hline gross total removal & $8(72.7 \%)$ & $17(50.0 \%)$ & \\
\hline subtotal removal + biopsy & $3(27.3 \%)$ & $14(41.2 \%)$ & \\
\hline unknown & $0(0 \%)$ & $3(8.8 \%)$ & \\
\hline RT field & & & 0.736 \\
\hline Small & $7(63.6 \%)$ & $19(55.9 \%)$ & \\
\hline Whole brain & $4(36.4 \%)$ & 15 (44.1\%) & \\
\hline EQD2 (Gy) & & & 0.987 \\
\hline Mean & 66.36 & 66.38 & \\
\hline SEM & 0.67 & 0.46 & \\
\hline
\end{tabular}

EQD2 equivalent dose in 2-Gy fractions, $O P$ operation, $R T$ radiotherapy

tumor bed boost or for tumor-site-only irradiation with a small field throughout the entire RT course (26 patients). For the latter, 11 patients were treated with a field-in field (FIF) boost protocol with RT that consisted of daily fractions of 180 cGy applied to a large target volume at the brain tumor site. This was followed by the subsequent application of 70 cGy (total 250 cGy daily) to a reduced field of the tumor bed, for a total dose of 6250-6750 cGy. The remaining 15 patients received conventional RT with 180-200 cGy per day. All RT protocols were administered in five fractions across the week.

For whole-brain irradiation, the photon beams at $6 \mathrm{MV}$ or $10 \mathrm{MV}$ were delivered via two-dimensional RT (2DRT). For tumor-site-only irradiation, three-dimensional conformal RT (3D-CRT) was used. The gross tumor volume (GTV) was defined according to the contrast-enhancing tumor on the CT or MRI images and included the residual tumor, perifocal edema, and entire resection cavity. The GTV plus a safe margin of $2 \mathrm{~cm}$ was the clinical target volume (CTV). In the FIF boost protocol, the CTV $+1 \mathrm{~cm}$ margin was irradiated as the large target volume, and the CTV alone was irradiated as the boost target volume. For 
3D-CRT, the CTV + $3 \mathrm{~mm}$ margin was the planning target volume (PTV) for daily setup variation. In the FIF boost protocol, the CTV $+1.3 \mathrm{~cm}$ margin was the PTV of the large target.

\section{Tissue microarray construction}

Areas showing the histopathologic features of GBM were selected on archival hemolysin and eosin (H\&E)-stained sections, and then representative areas of the tumor were marked on the corresponding paraffin block for tissue microarray (TMA) construction. Briefly, after the tissue cylinders were taken from the selected regions of the donor paraffin block, they were punched precisely into a recipient paraffin block using a tissue-arraying instrument. Multiple sections ( $1 \mu \mathrm{m}$ thick) were cut and mounted onto microscope slides. The TMA sections were evaluated by the pathologist (SLY), who did not know the outcomes of the patients.

\section{Immunohistochemistry (IHC)}

TMA sections were stained with an anti-galectin- 1 antibody (1:40, HPA000646; rabbit to human Sigma-Aldrich, St. Louis, MO) using a DAKO REAL EnVision (DAKO, Glostrup, Denmark) and an anti-galectin-3 antibody (Santa Cruz B2C10). The immunostaining patterns for galectin-1 and -3 in the intracellular tumor portion and extracellular stromal portion of the tissue samples were recorded. IHC staining was graded as no, weak, moderate, or strong staining according to the observed intensity (Fig. 1). The staining areas were calculated by the pathologist according to the staining percentage of all tumor cells in each TMA core.

The expression level of galectin-1 can be calculated by multiplying the amount of positively stained tumor cells (\%) by the staining intensity of immunoreactive tumor cells. The staining intensity of immunoreactive tumor cells was scored as follows: 0, no staining; 0.5, weak staining; 1, moderate staining; and 2, strong staining (Table 2).

We primarily used a single grader for the immunostaining results. To dramatically reduce intraobserver differences, reference to standard slides, which revealed the reference point of the staining score, was performed before each reading. The grader was completely unaware of the outcome of the patients, and thus bias of the scoring interpretation was negligible. Normal liver tissue was employed as a negative control for galectin staining was and melanoma as a positive control.

\section{Follow-up and statistics}

The first brain image analysis, MRI or CT, was obtained for all patients within 2-4 months after the completion of RT and then every 3-6 months or when clinically indicated, such as when new neurologic signs were observed. The optimal cutoffs of galectin- 1 and galectin-3 expression for median survival were estimated by univariate analysis with the log-rank test; those for OS, cancerspecific survival (CSS) and progression-free interval were estimated by multivariate analysis with a Cox regression model. OS and CSS were measured beginning on the first day of RT. OS was measured until the date of death from any cause or the last follow-up date, and CSS was defined as a survival measure representing cancer survival until the date of death (in the absence of another cause of death). The progression-free interval indicated the time interval from the first day of RT until the date of tumor progression, as confirmed by follow-up brain CT or MRI or by death.

\section{Results}

Univariate and multivariate analyses of treatment outcomes The median follow-up time was 12 months (range, 1.4207.0 months). The 3 -year OS was $11.1 \%$, and the median survival was 12 months. Multivariate analysis using galectin expression levels as a continuous variable revealed that the expression level of galectin-1 was independent of OS $(p=0.046)$. Fourteen of the 45 patients survived for more than two years. Each patient received an EQD2 $\geq 60$ Gy. Among these 14 patients, five patients received whole-brain irradiation followed by tumor bed boost and nine patients got tumor-site-only irradiation; of the latter, two were under the FIF boost protocol and seven conventional RT.

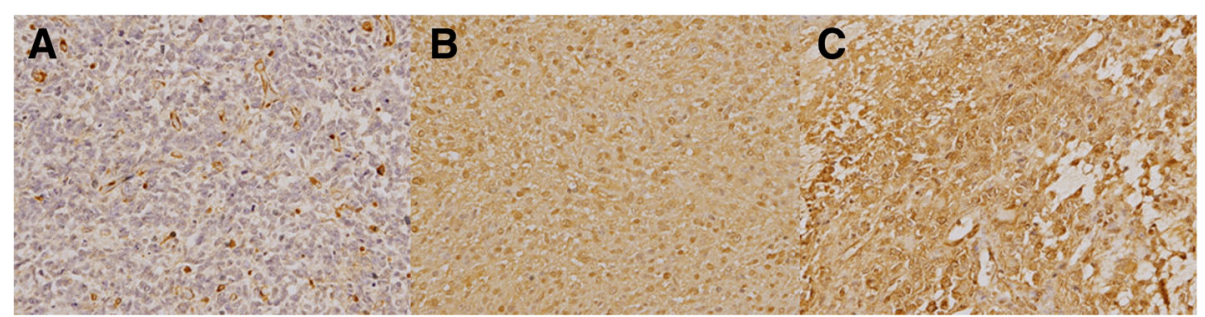

Fig. 1 Representative cases of galectin-1 immunostaining ( $\mathbf{a}, \mathbf{b}$, and $\mathbf{c}$, respectively). a Tumor cells show very faint staining, but the surrounding inflammatory cells and endothelial cells shows strong staining for galectin-1. Original magnification 200X. b Tumor cells show moderate nuclear staining. Original magnification 200X. c Tumor cells show diffuse strong nuclear staining. Original magnification 200X 
Table 2 Staining intensity scores

\begin{tabular}{lllll}
\hline Staining intensity & No staining & Weak & Moderate & Strong \\
\hline Score & 0 & 0.5 & 1 & 2 \\
\hline
\end{tabular}

We attempted to determine the optimal cutoffs of galectin- 1 and galectin- 3 expression levels using univariate analysis (Additional file 1). Patients with weak galectin-1 expression $(<35 \%)$ had a better median survival (27.9 months) than did those with strong ( $\geq 35 \%)$ galectin-1 expression (10.7 months; $p=0.009$ ). Among the 45 patients included, 34 showed strong galectin-1 expression and 11 weak galectin-1 expression. In addition, patients with weak galectin-3 expression $(<15 \%)$ had a better median survival (12.1 months) than did those with strong galectin-3 expression (10.7 months; $p=0.031)$. We compared characteristics between weak and strong galectin-1 expression (Table 1) and noted no significant difference. The 3-year OS was $27.3 \%$ and $5.9 \%(p=0.009)$ (Fig. 2) in patients with weak and strong galectin-1 expression, respectively, and the corresponding CSS was $27.3 \%$ and $7.6 \%$, respectively $(p=0.020)$ (Fig. 3 ).

We applied Cox regression to confirm the role of galectin-1 and again found it to be a significant factor of OS $(p=0.020 ; \mathrm{HR}=2.929 ; 95 \%$ CI $1.180-7.271)$ (Table 3). Other parameters, such as Eastern Cooperative Oncology Group (ECOG) performance, gender, surgical method, age $\geq 50$ years, tumor size $\geq$ $5 \mathrm{~cm}$, radiation field, and expression level of galectin3 , were not significant factors. CSS was also independent of galectin-1 overexpression $(p=0.022 ; \mathrm{HR}=$ $2.873 ; 95 \%$ CI 1.163-7.101) (Table 4), as was the progression-free rate $(p=0.037 ; \mathrm{HR}=6.080 ; 95 \% \mathrm{CI}$ 1.113-33.219) (Table 5).

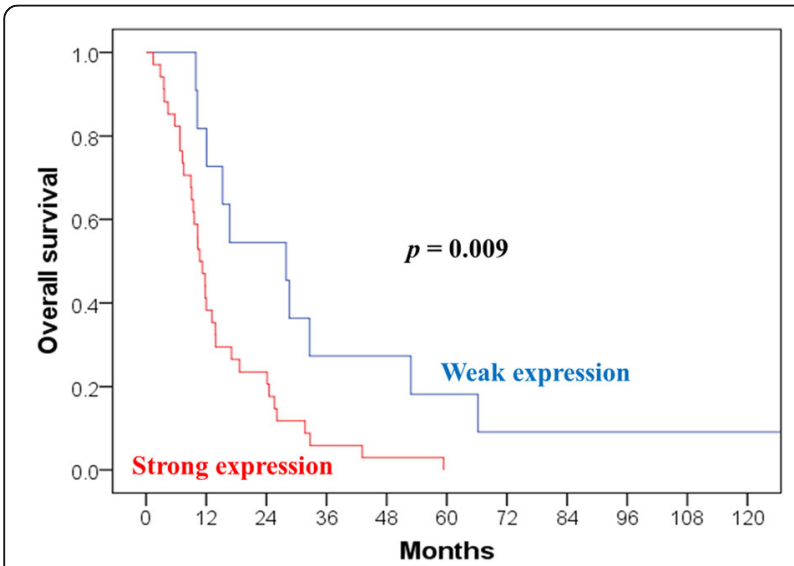

Fig. 2 Overall survival (OS) estimated according to the expression level of galectin-1. The expression level of galectin-1 was a significant factor of OS ( $p=0.009)$

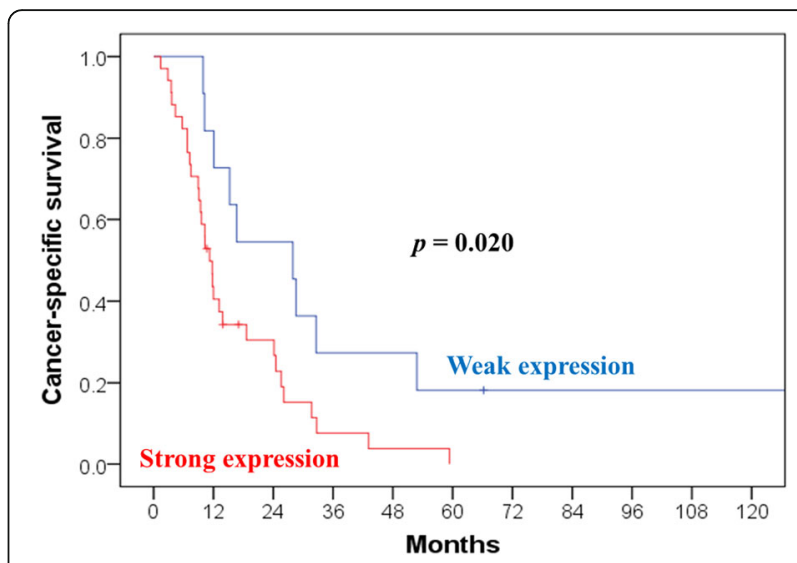

Fig. 3 Cancer-specific survival (CSS) estimated according to the expression level of galectin-1. The expression level of galectin-1 was a significant factor of $\operatorname{CSS}(p=0.020)$

\section{Discussion}

Although many molecular targets have been investigated in GBM, few studies have demonstrated the relationship between molecular targets and radiosensitivity. For example, epidermal growth factor receptor (EGFR) plays a role in GBM progression [20] but has no prognostic value in these patients [21]. In addition, EGFRvIII does not affect radiosensitivity in glioblastoma cells [22]. Moreover, the EGFR inhibitor gefitinib is not a radiosensitizer for newly diagnosed GBM [23]. Ras is downstream of EGFR, and k-ras mutations frequently occur in colon cancer. Indeed, ras may play a role in the angiogenic switch in astrocytomas [24] and be involved in chemoradioresistance [25]. TLN-4601, a k-ras inhibitor [26], cannot prevent GBM recurrence [27], and bevacizumab, an anti-vascular endothelial growth factor (VEGF) antibody, was found to be ineffective for treating GBM patients undergoing RT and TMZ in two recent randomized control trials $[28,29]$.

Galectin-1 is expressed in human gliomas and is associated with poor differentiation [13, 14]. It also functions

Table 3 MVA of OS

\begin{tabular}{lll}
\hline Parameters & $p$ value & HR (95\% Cl) \\
\hline Age $(\geq 50$ vs. $<50$ years) & 0.091 & $1.904(0.902-4.018)$ \\
Gender (male vs. female) & 0.907 & $1.045(0.501-2.179)$ \\
ECOG (3-4 vs 0-2) & 0.330 & $1.512(0.658-3.475)$ \\
Size ( $\geq 5$ cm vs. $<5$ cm) & 0.686 & $0.796(0.263-2.407)$ \\
OP method (non-gross total & 0.887 & $0.950(0.465-1.940)$ \\
vs. gross total) & & \\
RT field (whole brain vs. small) & 0.322 & $1.510(0.667-3.417)$ \\
Galectin-1 (strong vs. weak) & 0.020 & $2.929(1.180-7.271)$ \\
Galectin-3 (strong vs. weak) & 0.478 & $1.343(0.594-3.034)$ \\
\hline
\end{tabular}

MVA multivariate statistical analysis, OS overall survival, ECOG Eastern Cooperative Oncology Group, $O P$ operation, $R T$ radiotherapy 
Table 4 MVA of CSS

\begin{tabular}{lll}
\hline Parameter & $p$ value & HR $(95 \% \mathrm{Cl})$ \\
\hline Age ( $\geq 50$ vs. $<50$ years) & 0.140 & $1.791(0.826-3.883)$ \\
Gender (male vs. female) & 0.822 & $1.092(0.506-2.355)$ \\
ECOG (3-4 vs 0-2) & 0.591 & $1.273(0.527-3.075)$ \\
Size ( $\geq 5$ cm vs. $<5 \mathrm{~cm})$ & 0.701 & $0.792(0.241-2.603)$ \\
OP method (non-gross total & 0.627 & $0.831(0.393-1.755)$ \\
vs. gross total) & & \\
RT field (whole brain vs. small) & 0.382 & $1.449(0.631-3.325)$ \\
Galectin-1 (strong vs. weak) & 0.022 & $2.873(1.163-7.101)$ \\
Galectin-3 (strong vs. weak) & 0.993 & $1.004(0.426-2.364)$ \\
\hline
\end{tabular}

MVA multivariate statistical analysis, CSS cancer-specific survival, ECOG Eastern Cooperative Oncology Group, OP operation, $R T$ radiotherapy

downstream of the EGFR pathway through $\mathrm{H}$-ras by interacting and activating $\mathrm{H}$-ras to form guanosine triphosphate (GTP). In addition, galectin-1 is involved cancer progression, enhances the migration and invasion of human GBM cells [6, 7] and angiogenesis [8], inhibits the anti-tumor immunity of natural killer (NK) cells [30-32], is involved in glioma chemoresistance [33], and may be considered a biomarker because serum galectin1 levels are higher in patients with high-grade glioma than in healthy controls [10]. Despite evidence that galectin-1 may be involved in glioma progression in vitro, few animal studies on cancer progression have been performed $[7,13,14,31,32]$, and only one human study to date reports the role of galectin-1 in glioma prognosis. In this study, Rorive et al. compared the expression level of galectin-1 in high-grade astrocytic tumors from 41 patients ( 26 with GBM) who survived $<12$ months and > 24 months; the expression level of galectin-1 was significantly correlated with survival [13], but no details of treatment modality were reported. In the current study, galectin-1 overexpression was associated with poor survival and short time to progression following RT. To the best of our knowledge, the present study is the first report of the

Table 5 MVA of the progression-free rate

\begin{tabular}{lll}
\hline Parameter & $p$ value & HR (95\% Cl) \\
\hline Age ( $\geq 50$ vs. $<50$ years) & 0.159 & $2.369(0.712-7.878)$ \\
Gender (male vs. female) & 0.114 & $0.320(0.078-1.312)$ \\
ECOG (3-4 vs 0-2) & 0.258 & $2.292(0.544-9.650)$ \\
Size ( $\geq 5$ cm vs. $<5$ cm) & 0.819 & $1.213(0.232-6.350)$ \\
OP method (non-gross total & 0.023 & $7.408(1.320-41.585)$ \\
vs. gross total) & & \\
RT field (whole brain vs. small) & 0.609 & $0.716(0.202-2.535)$ \\
Galectin-1 (strong vs. weak) & 0.037 & $6.080(1.113-33.219)$ \\
Galectin-3 (strong vs. weak) & 0.089 & $0.181(0.025-1.299)$ \\
\hline
\end{tabular}

MVA multivariate statistical analysis, ECOG Eastern Cooperative Oncology Group, $O P$ operation, $R T$ radiotherapy association of galectin-1 with poor prognosis in GBM patients following RT alone. Thus, targeting galectin-1 has potential in treatment of GBM.

In the past, the entire brain was irradiated as treatment for GBM because malignant gliomas may spread along white matter tracts [34-37]. Subsequent studies found that GBM usually recurs within $2 \mathrm{~cm}$ of the initial tumor volume [38, 39], and histologic analysis has shown that perifocal edema often corresponds to parenchyma infiltrated by isolated tumor cells [40]. Therefore, local irradiation, rather than whole-brain RT (WBRT), is the standard radiation treatment for GBM. Because our study was limited by early data prior to 2002, some patients had still been treated with WBRT. In GBM patients, mortality largely occurs due to local recurrence or progression in or adjacent to the resection cavity, as opposed to extracranial metastases. Although the radiation groups included in this study were inhomogeneous, we considered that the local dose is key for outcomes. Accordingly, to exclude an RT regimen effect, we used EQD2 to exclude patients who had received a local dose under $60 \mathrm{~Gy}$.

Currently, the standard treatment for GBM is RT combined with TMZ [4]. Because radiation can induce galectin-1 expression in glioma cells [41], inhibition of galectin-1 expression is important for RT. Danhier et al. reported in an animal study that knockdown of galectin1 and EGFR using nanocapsules can decrease TMZ resistance in glioblastoma [42]. TMZ also effectively knocks down galectin-1 $[9,43]$. Therefore, the combination of TMZ and RT may be successful therapy.

The median survival of our GBM patients was 12.0 months, which was comparable to that in the European Organisation for Research and Treatment of Cancer - National Cancer Institute Canada (EORTC/NCIC) trial (12.1 months) [4]. In patients with and without MGMT methylation and undergoing RT alone, the median survival was 15.3 and 11.8 months [5], respectively. Although TMZ significantly improved the median survival in patients with MGMT methylation (from 15.3 to 21.7 months), this was not observed in those without MGMT methylation (from 11.8 to 12.7 months). In the present study, the median survival of patients with galectin-1 overexpression was 10.7 months, which is very similar to the median survival of patients without MGMT methylation. Despite a median survival of 27.9 months in patients with weak galectin-1 expression, the proportion of this favorable group was low, at $32.4 \%$. Due to the lack of test reagents in our hospital, we failed to obtain the MGMT promotor methylation status, which may affect the efficacy of TMZ. In this study, all the patient received brain RT only without planned concurrent nor adjuvant TMZ. Further analysis of the 
effect of TMZ in patients with weak and strong galectin-1 expression is encouraged.

Galectin-3 is a common molecule studied in cancer research, especially in colon cancer, whereas only one study [44] involving an animal model has reported that galectin-3 is associated with GBM progression. Regardless, our results do not prove that galectin-3 is a prognostic factor compared with galectin-1. Galectin-1 and galectin-3 are associated with $\mathrm{H}$-ras [45] and K-ras [46] signals, respectively, and $\mathrm{H}$-ras [24, 47-49] but not K-ras is involved in GBM progression. Therefore, further in vitro and in vivo research is encouraged to determine the roles of galectin-1 and - 3 in GBM radioresistance.

The limitations of this study are its retrospective nature and limited sample size. According to the national law of human research, informed consent for research should be obtained for work performed in our country after 2002 . As we could only include patients before 2002, and the sample size was therefore small. Because these patients were treated a long time ago, almost all were dead, their charts were destroyed, and the time to progression could not be confirmed. Nonetheless, the time to progression and the survival time were correlated in patients who underwent imaging follow-up; not all patients were evaluated for time to progression, yet galectin-1 remained a significant factor of GBM progression after RT. In addition, MGMT promoter methylation is associated with patient response to alkylating agents and OS. Because the proportion of MGMT promoter methylation in the included patients was undetermined, it remains unclear whether galectin-1 expression may affect the importance of the MGMT promoter methylation status for prognosis. Future studies are required to analyze the effect of other factors, such as MGMT methylation promoter status.

\section{Conclusions}

Ggalectin-1 expression is a poor prognostic factor for patients with GBM treated with adjuvant RT. Studies investigating the targeting of galectin-1 for radioresistance are encouraged in an effort to treat this aggressive brain tumor.

\section{Additional file}

Additional file 1: Optimal cutoff of galectin-1 and galectin-3 for median survival (month). (DOC 38 kb)

\section{Abbreviations}

2DRT: Two-dimensional RT; 3D-CRT: Three-dimensional conformal RT; CSS: Cancerspecific survival; CTV: Clinical target volume; ECOG: Eastern Cooperative Oncology Group; EGFR: Epidermal growth factor receptor; EQD2: Equivalent dose in 2-Gy fractions; FIF: Field-in-field; GBM: Glioblastoma multiforme; GTP: Guanosine triphosphate; GTV: Gross tumor volume; MGMT: O(6)-methylguanine-DNA methyltransferase; OS: Overall survival; PTV: Planning target volume; RT: Radiotherapy; TMA: Tissue microarray; TMZ: Temozolomide; VEGF: Vascular endothelial growth factor

\section{Acknowledgements}

Not applicable.

\section{Funding}

This work was partially supported by a grant from the Ministry of Science and Technology Research Project (103-2314-B-182A-065-MY2).

The funding body had no role in the design of the study and collection, analysis, and interpretation of data and in writing the manuscript.

\section{Availability of data and materials}

The datasets used and/or analyzed during the current study are available from the corresponding author upon reasonable request.

\section{Authors' contributions}

SYC and EYH analyzed and collected the patient data and were major contributors in writing the manuscript. SLY and CCH performed the microarray examination of the tissue. All authors read and approved the final manuscript.

\section{Ethics approval and consent to participate}

This study was approved by the Institutional Review Board of Chang Gung Memorial Hospital (102-5087B).

Consent for publication

Not applicable.

\section{Competing interests}

The authors declare that they have no competing interests.

\section{Publisher's Note}

Springer Nature remains neutral with regard to jurisdictional claims in published maps and institutional affiliations.

\section{Author details}

'Departments of Radiation Oncology, Kaohsiung Chang Gung Memorial Hospital, 123 Ta-Pei Road, Niao-Song Dist, Kaohsiung City 83301, Taiwan. ${ }^{2}$ Department of Pathology, Kaohsiung Chang Gung Memorial Hospital, Chang Gung University College of Medicine, Hospital, 123 Ta-Pei Road, Niao-Song Dist, Kaohsiung City 83301, Taiwan. ${ }^{3}$ Department of Radiation Oncology, Xiamen Chang Gung Hospital, No. 123, Xiafei Rd., Haicang District, Fujian, China. ${ }^{4}$ School of Traditional Chinese Medicine, Chang Gung University College of Medicine, No. 259, Wenhua 1st Rd., Guishan Dist., Taoyuan City, Taiwan. ${ }^{5}$ Department of Pathology, An Nan Hospital, China Medical University, No. 66, Sec.2, Changhe Road, Annan Dist, Tainan City 709, Taiwan. ${ }^{6}$ Department of Radiation Oncology, Kaohsiung Chang Gung Memorial Hospital, 123 Ta-Pei Road, Niao-Song Dist, Kaohsiung City 83301, Taiwan.

Received: 2 May 2017 Accepted: 23 January 2018

Published online: 30 January 2018

\section{References}

1. Stark AM, Nabavi A, Mehdorn HM, Blomer U. Glioblastoma multiforme-report of 267 cases treated at a single institution. Surg Neurol. 2005;63(2):162-9. discussion 169

2. Dobes M, Khurana VG, Shadbolt B, Jain S, Smith SF, Smee R, Dexter M, Cook R. Increasing incidence of glioblastoma multiforme and meningioma, and decreasing incidence of schwannoma (2000-2008): findings of a multicenter Australian study. Surg Neurol Int. 2011;2:176

3. Lacroix M, Abi-Said D, Fourney DR, Gokaslan ZL, Shi W, DeMonte F, Lang FF, McCutcheon IE, Hassenbusch SJ, Holland E, et al. A multivariate analysis of 416 patients with glioblastoma multiforme: prognosis, extent of resection, and survival. J Neurosurg. 2001;95(2):190-8.

4. Stupp R, Mason WP, Van Den Bent MJ, Weller M, Fisher B, Taphoorn MJ, Belanger K, Brandes AA, Marosi C, Bogdahn U, et al. Radiotherapy plus concomitant and adjuvant temozolomide for glioblastoma. N Engl J Med. 2005;352(10):987-96.

5. Hegi ME, Diserens AC, Gorlia T, Hamou MF, de Tribolet N, Weller M, Kros JM, Hainfellner JA, Mason W, Mariani L, et al. MGMT gene silencing and benefit from temozolomide in glioblastoma. N Engl J Med. 2005;352(10):997-1003. 
6. Jung TY, Jung S, Ryu HH, Jeong YI, Jin YH, Jin SG, Kim IY, Kang SS, Kim HS. Role of galectin-1 in migration and invasion of human glioblastoma multiforme cell lines. J Neurosurg. 2008;109(2):273-84.

7. Toussaint LG 3rd, Nilson AE, Goble JM, Ballman KV, James CD, Lefranc F, Kiss R, Uhm JH. Galectin-1, a gene preferentially expressed at the tumor margin, promotes glioblastoma cell invasion. Mol Cancer. 2012;11:32.

8. Thijssen VL, Postel R, Brandwijk RJ, Dings RP, Nesmelova I, Satijn S, Verhofstad N, Nakabeppu Y, Baum LG, Bakkers J, et al. Galectin-1 is essential in tumor angiogenesis and is a target for antiangiogenesis therapy. Proc Natl Acad Sci U S A. 2006;103(43):15975-80.

9. Le Mercier M, Mathieu V, Haibe-Kains B, Bontempi G, Mijatovic T, Decaestecker C, Kiss R, Lefranc F. Knocking down galectin 1 in human hs683 glioblastoma cells impairs both angiogenesis and endoplasmic reticulum stress responses. J Neuropathol Exp Neurol. 2008;67(5):456-69.

10. Verschuere T, Van Woensel M, Fieuws S, Lefranc F, Mathieu V, Kiss R, Van Gool SW, De Vleeschouwer S. Altered galectin-1 serum levels in patients diagnosed with high-grade glioma. J Neuro-Oncol. 2013;115(1):9-17.

11. Huang EY, Chen YF, Chen YM, Lin IH, Wang CC, Su WH, Chuang PC, Yang $\mathrm{KD}$. A novel radioresistant mechanism of galectin-1 mediated by H-Rasdependent pathways in cervical cancer cells. Cell Death Dis. 2012;3:e251.

12. Huang EY, Chanchien CC, Lin H, Wang CC, Wang CJ, Huang CC. Galectin-1 is an independent prognostic factor for local recurrence and survival after definitive radiation therapy for patients with squamous cell carcinoma of the uterine cervix. Int J Radiat Oncol Biol Phys. 2013;87(5):975-82.

13. Rorive S, Belot N, Decaestecker C, Lefranc F, Gordower L, Micik S, Maurage CA, Kaltner H, Ruchoux MM, Danguy A, et al. Galectin-1 is highly expressed in human gliomas with relevance for modulation of invasion of tumor astrocytes into the brain parenchyma. Glia. 2001;33(3):241-55.

14. Camby I, Belot N, Rorive S, Lefranc F, Maurage CA, Lahm H, Kaltner H, Hadari Y, Ruchoux MM, Brotchi J, et al. Galectins are differentially expressed in supratentorial pilocytic astrocytomas, astrocytomas, anaplastic astrocytomas and glioblastomas, and significantly modulate tumor astrocyte migration. Brain pathology (Zurich, Switzerland). 2001;11(1):12-26.

15. Tribius S, Pidel A, Casper D. ATM protein expression correlates with radioresistance in primary glioblastoma cells in culture. Int J Radiat Oncol Biol Phys. 2001;50(2):511-23.

16. Hirota $Y$, Masunaga S, Kondo N, Kawabata S, Hirakawa H, Yajima H, Fujimori A, Ono K, Kuroiwa T, Miyatake S. High linear-energy-transfer radiation can overcome radioresistance of glioma stem-like cells to low linear-energytransfer radiation. J Radiat Res. 2014;55(1):75-83.

17. Bleehen NM, Stenning SP. A Medical Research Council trial of two radiotherapy doses in the treatment of grades 3 and 4 astrocytoma. The Medical Research Council brain tumour working party. Br J Cancer. 1991;64(4):769-74.

18. Curran WJ Jr, Scott CB, Horton J, Nelson JS, Weinstein AS, Fischbach AJ Chang $\mathrm{CH}$, Rotman M, Asbell SO, Krisch RE, et al. Recursive partitioning analysis of prognostic factors in three radiation therapy oncology group malignant glioma trials. J Natl Cancer Inst. 1993:85(9):704-10.

19. Jeremic B, Shibamoto Y, Grujicic D, Milicic B, Stojanovic M, Nikolic N, Dagovic A, Aleksandrovic J. Short-course radiotherapy in elderly and frail patients with glioblastoma multiforme. A phase II study. J Neuro-Oncol. 1999; 44(1):85-90.

20. Fan QW, Cheng CK, Gustafson WC, Charron E, Zipper P, Wong RA, Chen J, Lau J, Knobbe-Thomsen C, Weller M, et al. EGFR phosphorylates tumorderived EGFRvIII driving STAT3/5 and progression in glioblastoma. Cancer Cell. 2013;24(4):438-49.

21. Chakravarti A, Seiferheld W, Tu X, Wang H, Zhang HZ, Ang KK, Hammond E, Curran W Jr, Mehta M. Immunohistochemically determined total epidermal growth factor receptor levels not of prognostic value in newly diagnosed glioblastoma multiforme: report from the radiation therapy oncology group. Int J Radiat Oncol Biol Phys. 2005;62(2):318-27.

22. Struve N, Riedel M, Schulte A, Rieckmann T, Grob TJ, Gal A, Rothkamm K, Lamszus K, Petersen C, Dikomey E, et al. EGFRvill does not affect radiosensitivity with or without gefitinib treatment in glioblastoma cells. Oncotarget. 2015;6(32): 33867-77.

23. Chakravarti A, Wang M, Robins HI, Lautenschlaeger T, Curran WJ, Brachman DG, Schultz CJ, Choucair A, Dolled-Filhart M, Christiansen J, et al. RTOG 0211: a phase $1 / 2$ study of radiation therapy with concurrent gefitinib for newly diagnosed glioblastoma patients. Int J Radiat Oncol Biol Phys. 2013;85(5):1206-11.

24. Feldkamp MM, Lau N, Rak J, Kerbel RS, Guha A. Normoxic and hypoxic regulation of vascular endothelial growth factor (VEGF) by astrocytoma cells is mediated by Ras. Int J Cancer. 1999;81(1):118-24.
25. Chakravarti A, Chakladar A, Delaney MA, Latham DE, Loeffler JS. The epidermal growth factor receptor pathway mediates resistance to sequential administration of radiation and chemotherapy in primary human glioblastoma cells in a RAS-dependent manner. Cancer Res. 2002;62(15):4307-15.

26. Campbell PM, Boufaied N, Fiordalisi JJ, Cox AD, Falardeau P, Der CJ, Gourdeau H. TLN-4601 suppresses growth and induces apoptosis of pancreatic carcinoma cells through inhibition of Ras-ERK MAPK signaling. J Mol Signal. 2010;5:18

27. Mason WP, Belanger K, Nicholas G, Vallieres I, Mathieu D, Kavan P, Desjardins A, Omuro A, Reymond D. A phase II study of the Ras-MAPK signaling pathway inhibitor TLN-4601 in patients with glioblastoma at first progression. J NeuroOncol. 2012:107(2):343-9.

28. Gilbert MR, Dignam JJ, Armstrong TS, Wefel JS, Blumenthal DT, Vogelbaum MA, Colman $\mathrm{H}$, Chakravarti A, Pugh S, Won M, et al. A randomized trial of bevacizumab for newly diagnosed glioblastoma. N Engl J Med. 2014;370(8):699-708.

29. Chinot OL, Wick W, Mason W, Henriksson R, Saran F, Nishikawa R, Carpentier AF, Hoang-Xuan K, Kavan P, Cernea D, et al. bevacizumab plus radiotherapytemozolomide for newly diagnosed glioblastoma. N Engl J Med. 2014; 370(8):709-22.

30. Lowenstein PR, Baker GJ, Castro MG. Cracking the glioma-NK inhibitory code: toward successful innate immunotherapy. Oncoimmunology. 2014; 3(11):e965573.

31. Baker GJ, Chockley P, Yadav VN, Doherty R, Ritt M, Sivaramakrishnan S, Castro MG, Lowenstein PR. Natural killer cells eradicate galectin-1deficient glioma in the absence of adaptive immunity. Cancer Res. 2014;74(18):5079-90.

32. Verschuere T, Toelen J, Maes W, Poirier F, Boon L, Tousseyn T, Mathivet T, Gerhardt H, Mathieu V, Kiss R, et al. Glioma-derived galectin-1 regulates innate and adaptive antitumor immunity. Int J Cancer. 2014:134(4):873-84.

33. Le Mercier M, Lefranc F, Mijatovic T, Debeir O, Haibe-Kains B, Bontempi G, Decaestecker C, Kiss R, Mathieu V. Evidence of galectin-1 involvement in glioma chemoresistance. Toxicol Appl Pharmacol. 2008;229(2):172-83.

34. Kramer $\mathrm{S}$. Tumor extent as a determining factor in radiotherapy of glioblastomas. Acta Radiol Ther Phys Biol. 1969;8(1-2):0567-8064. (Print)):111-117

35. Bull JWDRR. The radiographic localization of intracerebral gliomata. J Fac Radiol Lond. 1957:8:147-57.

36. WE. D. Removal of right cerebral hemisphere for certain tumors with hemiplegia. JAMA. 1928;90:823-5.

37. Matsukado Y Fau - Maccarty CS, Maccarty CS Fau - Kernohan JW, Kernohan JW: The growth of glioblastoma multiforme (astrocytomas, grades 3 and 4) in neurosurgical practice. Neurosurg 1961, 18(0022-3085 (Print)):636-644.

38. Hochberg Fh Fau - Pruitt A, Pruitt A: Assumptions in the radiotherapy of glioblastoma. Neurology 1980, 30(0028-3878 (Print)):907-911.

39. Wallner KE, Galicich Jh Fau - Krol G, Krol G Fau - Arbit E, Arbit E Fau - Malkin MG, Malkin MG: Patterns of failure following treatment for glioblastoma multiforme and anaplastic astrocytoma. International journal of radiation oncology, biology, physics 1989, 16(0360-3016 (Print)):1045-1409.

40. Kelly Pj Fau - Daumas-Duport C, Daumas-Duport C Fau - Scheithauer BW, Scheithauer Bw Fau - Kall BA, Kall Ba Fau - Kispert DB, Kispert DB: Stereotactic histologic correlations of computed tomography- and magnetic resonance imaging-defined abnormalities in patients with glial neoplasms. Mayo Clinic Proc 1987, 62(0025-6196 (Print)):450-459.

41. Strik HM, Schmidt K, Lingor P, Tonges L, Kugler W, Nitsche M, Rabinovich GA, Bahr M. Galectin-1 expression in human glioma cells: modulation by ionizing radiation and effects on tumor cell proliferation and migration. Oncol Rep. 2007;18(2):483-8.

42. Danhier F, Messaoudi K, Lemaire L, Benoit JP, Lagarce F. Combined antiGalectin-1 and anti-EGFR siRNA-loaded chitosan-lipid nanocapsules decrease temozolomide resistance in glioblastoma: in vivo evaluation. Int J Pharm. 2015; 481(1-2):154-61

43. Messaoudi K, Clavreul A, Lagarce F. Toward an effective strategy in glioblastoma treatment. Part l: resistance mechanisms and strategies to overcome resistance of glioblastoma to temozolomide. Drug Discov Today. 2015;20(7):899-905.

44. Ikemori RY, Machado CM, Furuzawa KM, Nonogaki S, Osinaga E, Umezawa K, de Carvalho MA, Verinaud L, Chammas R. Galectin-3 up-regulation in hypoxic and nutrient deprived microenvironments promotes cell survival. PLoS One. 2014:9(11):e111592.

45. Paz A, Haklai R, Elad-Sfadia G, Ballan E, Kloog Y. Galectin-1 binds oncogenic $\mathrm{H}$-Ras to mediate Ras membrane anchorage and cell transformation. Oncogene. 2001;20(51):7486-93. 
46. Shalom-Feuerstein R, Plowman SJ, Rotblat B, Ariotti N, Tian T, Hancock JF, Kloog Y. K-ras nanoclustering is subverted by overexpression of the scaffold protein galectin-3. Cancer Res. 2008;68(16):6608-16.

47. Bunda S, Burrell K, Heir P, Zeng L, Alamsahebpour A, Kano Y, Raught B, Zhang ZY, Zadeh G, Ohh M. Inhibition of SHP2-mediated dephosphorylation of Ras suppresses oncogenesis. Nat Commun. 2015;6:8859.

48. Kimmelman AC, Qiao RF, Narla G, Banno A, Lau N, Bos PD, Nunez Rodriguez N, Liang BC, Guha A, Martignetti JA, et al. Suppression of glioblastoma tumorigenicity by the Kruppel-like transcription factor KLF6. Oncogene. 2004;23(29):5077-83.

49. Sonoda Y, Ozawa T, Aldape KD, Deen DF, Berger MS, Pieper RO. Akt pathway activation converts anaplastic astrocytoma to glioblastoma multiforme in a human astrocyte model of glioma. Cancer Res. 2001; 61(18):6674-8

Submit your next manuscript to BioMed Central and we will help you at every step:

- We accept pre-submission inquiries

- Our selector tool helps you to find the most relevant journal

- We provide round the clock customer support

- Convenient online submission

- Thorough peer review

- Inclusion in PubMed and all major indexing services

- Maximum visibility for your research

Submit your manuscript at www.biomedcentral.com/submit
Biomed Central 